\title{
The influence of camouflage and prey type on predatory decisions of jumping spider
}

\author{
MACIEJ BARTOS \\ Department of Teacher Training and Biological Diversity Studies, University of Lodz, Banacha 1/3, 90-237 Lodz, Poland \\ E-mail: bartos@biol.uni.lodz.pl
}

\section{Abstract}

Decisions made by predators during predatory encounters are often based on multiple factors that may influence the outcome of the encounters. For stalking predators their visibility to the prey and the ability of their prey to escape may be important factors influencing predatory success. Hence they are likely to adapt their predatory behavior when approaching prey on backgrounds with different camouflaging properties, but only if the prey is able to escape. To test whether jumping spiders flexibly adapt their predatory behavior to camouflaging properties of the background and prey type, the behavior of Yllenus arenarius (Araneae, Salticide), a cryptically colored jumping spider hunting leafhoppers (high escape potential) and caterpillars (low escape potential) on two types of background: matching and non-matching for the spiders was analyzed. Background color had a significant effect on the spiders' jumping distance and their predatory success, but only if the prey had a high escape potential. No differences occurred between backgrounds if the prey could not escape. On camouflaging background the spiders attacked leafhoppers from a shorter distance and had a higher success than on non-camouflaging background.

KEY WORDS: crypsis, predatory behavior, behavioral plasticity, salticid spider, Yllenus arenarius

\section{Introduction}

In recent decades it has become increasingly apparent that predatory behavior of jumping spiders is complex and flexible (Jakob et al. 2011; Nelson \& Jackson 2011). Decisions the spiders make during predatory encounters are often based on multiple factors that may influence the outcome of the encounter. Jumping spiders have been reported to adapt their predatory behavior to various properties of their prey, such as the potential of the prey to escape (Edwards \& Jackson 1993; Bear \& Hasson 1997; Bartos 2007), the ability of the prey to detect the spider (Bear \& Hasson 1997; Li et al. 2003) or to injure the spider (Li et al. 1999; Jackson \& Carter 2001). Such dangerous prey can be approached differently when it is capable of attacking the spider or when its ability to defend itself is impaired (Li \& Jackson 2003). Some communal jumping spiders of the genus Portia make especially intricate predatory decisions based on the presence or absence of their prey nest, the identity of spiders inside and outside the nest and the position of these spiders relative to each other at the nest (Jackson \& Nelson 2012). Making decisions requires from the spiders visual assessment of their environment and visual prey identification, often from a distance, and jumping spiders, due to their unique eyes, possess such abilities. 
Jumping spiders are typical day hunters with well developed eyes (Land 1969a, b). They have four pairs of simple eyes (Forster 1982). Three pairs of these eyes are relatively small 'secondary eyes' and function primarily as movement detectors (Land 1972, 1985), but may also be used in depth perception and initial categorization of moving objects (Zurek \& Nelson 2012). One pair of large forward-facing 'principal eyes' is positioned at the front of the cephalothorax. Principal eyes possess a unique structure (Land 1969a, b; Blest et al. 1990) and provide spatial acuity unparalleled among any terrestrial invertebrates (Williams \& McIntyre 1980; Harland \& Jackson 2004). Some of the spiders can discriminate between objects spaced $0.12 \mathrm{~mm}$ apart from a distance of about $200 \mathrm{~mm}$ (Harland \& Jackson 2004), which enables them to identify their prey based on a high degree of detail (Jackson \& Nelson 2012; Nelson \& Jackson 2012a, b). Jumping spiders can discern green, blue and ultraviolet (Land 1969a; Yamashita \& Tateda 1976; Peaslee \& Wilson 1989; Blest et al. 1981) and were reported to discriminate between differently colored backgrounds (Nakamura \& Yamashita 2000).

Jumping spiders are stalking predators. They do not build prey-capture webs, but instead they usually stalk their prey. A typical jumping spider's predatory sequence begins when the spider detects a moving object in its neighborhood. Detection is followed by orientation towards the object and identification of such an object as prey or non-prey. If the object is identified as prey, the spider reduces the distance to it initially by a quick run and later, when close to the prey, by a slow walk and stalk. Finally the spider strikes the prey from a certain distance (Forster 1977).

During approach a stalking predator has to make significant decisions, e.g. about the direction, the speed of approach and the distance from which it can attack its prey. Different predatory decisions are associated, however, with different types of risk that may affect the outcome of the encounter (Bear \& Hasson 1997). A stalking predator may fail if its prey runs or flies away even without perceiving the predator (spontaneous departure), if the prey perceives the predator and escapes before the strike (early detection), if the prey escapes during or after the strike (escape) and finally, if predatory sequence is interrupted by another predator or the spider's own enemy (interference). The analysis of all the potential risks reveals numerous trade-offs between contradictory decisions, each of which is associated with a different pay-off (Bear \& Hasson 1997). For example, quick approach reduces the risk of prey's spontaneous departure and the risk of interference by other predators, but it increases the risk of predator's detection. Close approach reduces the risk of imprecise strike, but again increases the risk of predator's detection. We can assume that every factor decreasing the probability of predator's detection, such as camouflage, should change predator's decision toward the behaviors decreasing the other risks and should possibly influence predatory success. Hence, we can expect that on camouflaging background predators will attack from a shorter distance and have higher predatory success than on a non-camouflaging background. In only one study, where stalking predator's decisions were analyzed, it has been shown that a jumping spider, Plexippus paykulli, adapts its hunting behavior to its visibility to the prey and the ability of its prey to escape (Bear \& Hasson 1997). On noncamouflaging background $P$. paykulli approached flies with higher velocities than on camouflaging background. The spider attacked the prey from longer distances on non-camouflaging than on camouflaging background. The effect of background was absent, however, when the prey were fly maggots.

The aim of this study is to check if a cryptically colored jumping spider, Yllenus arenarius, adapts its predatory behavior to its own visibility to the prey and to prey escape potential. This study is similar in some aspects to the study by Bear and Hasson (1997) by testing a similar problem. The study, however, uses a different model 
(a highly cryptic salticid) and different prey (leafhoppers and caterpillars). Another difference is the use of living prey instead of dead prey. This enables checking how a predator's decisions affect predatory success and prey-specific behaviors, which has not been tested before.

Yllenus arenarius, a jumping spider used in this study, seems to be a particularly suitable model to test the influence of predator's visibility on its predatory decisions, because the spider is a cryptically colored stalking predator. The natural habitats of $Y$. arenarius are bare sandy areas providing very few

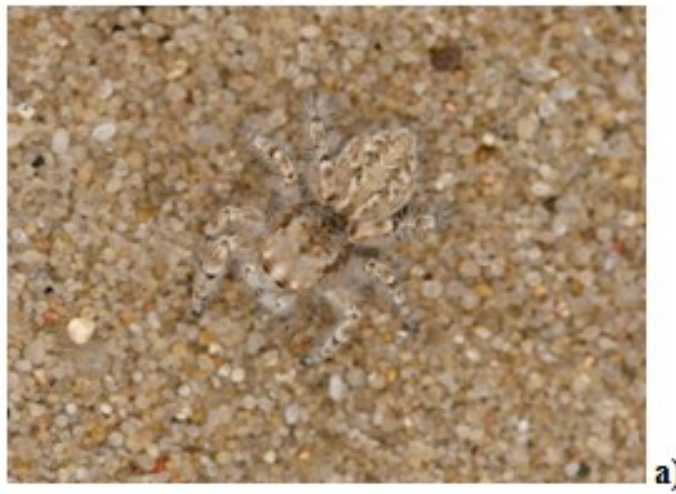

hiding places, generally not exploited by the spiders as hunting sites. Instead, the spiders await their prey in the open, non vegetated areas, where their highly cryptic coloration provides camouflage on the sand surface (Fig. 1a). There are two major substrates occurring in the natural habitat of $Y$. arenarius: light areas of lose sand, camouflaging for juveniles, and dark patches of brown sand, which are non-camouflaging for juveniles. In this study the spiders were tested on the backgrounds possessing similar camouflaging properties to those found in the spiders' natural habitat.

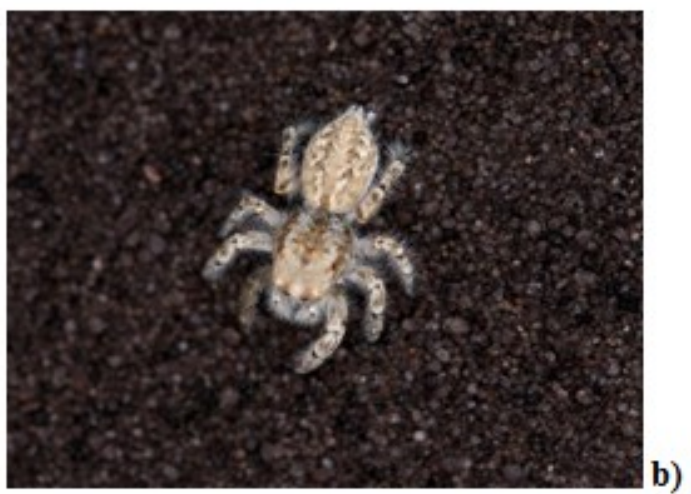

Figure 1. Yllenus arenarius on two backgrounds used in the experiments: a) light background, b) dark background.

\section{Methods}

The spiders used in the experiments were one-year-old juveniles of Yllenus arenarius. The spiders' age was determined based on their phenology, size, and maturity according to a previously developed method (Bartos 2005). All the spiders were collected from a dune in Central Poland (Kwilno, $51^{\circ} 59^{\prime} \mathrm{N}$, $19^{\circ} 30^{\prime} \mathrm{E}$ ). In order to reduce the influence of laboratory conditions on the behavior of $Y$. arenarius the experiments were carried out the same day or the day after the spiders were collected. Before the experiments the spiders were kept individually in glass containers $(1000 \mathrm{ml})$ with a layer of dune sand on the bottom. After finishing the experiments the spiders were released in the field. In order to avoid using the same spiders more than once the spiders were released in the areas of the dune that were effectively isolated by dense vegetation from those areas where the spiders were later collected for the experiments.
Two insect taxa with different abilities to escape were chosen as prey animals. The leafhoppers of the genus Arocephalus (Hemiptera, Cicadellidae) (body length: 3.5$4 \mathrm{~mm}$ ) were used as the prey with high escape potential. The caterpillars of Pyralis farinalis (Lepidoptera, Pyralidae) (body length: 6-7 $\mathrm{mm}$ ) were used as the prey with low escape potential. Leafhoppers, including those from the genus Arocephalus, are common in the natural diet of Y. arenarius (Bartos 2011). The caterpillars of $P$. farinalis were not reported in the spider's natural diet, but the spider was found to capture the caterpillars of other lepidopteran species (Bartos 2004, 2011). The spiders were observed to use preyspecific predatory behavior against leafhoppers and caterpillars (Bartos 2007, 2008). Leafhoppers were collected in the field by sweep-netting dune grass on the day of the experiment or the day before and they were 
held individually in plastic tubes. In order to reduce mortality of the prey, the insects were stored in a refrigerator at $5^{\circ} \mathrm{C}$ and they were taken out $15 \mathrm{~min}$ before the experiment started. Caterpillars were obtained from a lab culture. Each prey item was chosen randomly for the experiments.

The experiments were carried out in a white cardboard arena $(15 \mathrm{~cm}$ high by $20 \mathrm{~cm}$ diameter) with a $1 \mathrm{~cm}$-thick layer of sand on the bottom. Two types of backgrounds were used (Fig. 1): light background (dune sand camouflaging for the spiders), and dark background (dune sand dyed dark brown, non-camouflaging for the spiders). The sand was dyed with a brown food dye non-toxic for spiders and their prey.

Spider camouflage was judged visually. In order to reduce a possible influence of UV light, to which some insects and spiders are sensitive (Yamashita \& Tateda 1976; Peaslee \& Wilson 1989; Briscoe \& Chittka 2001), and which is not perceived by the human eye, only artificial light sources with very low intensity of UV light (incandescent bulb) or emitting UV-C in spectra not detected by insects and jumping spiders (Li et al. 2008) (fluorescent tube ceiling lights emitting UV waves around $254 \mathrm{~nm}$ ) were used in the lab. Because the spiders were tested on highly contrasting or matching backgrounds illuminated with high intensity of visible light it appears unlikely that such low intensity of UV light produced by the light sources could have a significant effect on the overall visibility of the tested spiders.

Each spider was chosen randomly for the tests and it was used only once in the whole set of experiments. The spider was first dropped onto the sand and after ten seconds a prey item was introduced about eight $\mathrm{cm}$ from the spider. The prey and the spider were dropped through non-transparent plastic tubes. The tube used to drop the prey was removed only when the prey stopped moving and remained motionless for $10 \mathrm{sec}$. The prey was left with the spider for $5 \mathrm{~min}$ and their interactions were recorded with a camera placed above the arena. In order to exclude a possibility that the spiders' reactions resulted from the activity patterns of their prey on different backgrounds all the instances when the prey moved during the spider's approach were excluded from the analysis. The fraction of excluded data was $25 \%$ or less and it was similar irrespective of the background. From the tests with leafhoppers on light background 6 of 24 trials were excluded and on dark background 5 of 23 were excluded. Sand surface was brushed between the tests to remove draglines and after that the surface layer (about $5 \mathrm{~mm}$-thick) was removed. The arena was then refilled with new sand up to the previous level. All the experiments were carried out between 09:00 and 16:00 (laboratory light regime, $12 \mathrm{~L}: 12 \mathrm{D}$, lights on at 08:00). Lighting was from a $100 \mathrm{~W}$ PILA incandescent bulb positioned $0.5 \mathrm{~m}$ above the arena and by fluorescent tube ceiling lights 2 $\mathrm{m}$ above the arena.

In each encounter the spider's predatory success was recorded and the jumping distance was measured. The distance was measured in Corel Draw 9.0 with a millimeter scale recorded together with the hunting sequence. Measurements were made in screen captures. The occurrence of prey-specific predatory behaviors: stalk, frontal approach and jump away (Bartos 2007), was also recorded.

The influence of background color was tested independently in approach to leafhoppers and in approach to caterpillars. Jumping distance was tested with t-test $\left(t_{\mathrm{n}}\right)$ and differences in the frequencies of preyspecific behaviors were tested with G-test $\left(G_{\mathrm{df} ; \mathrm{n}}\right)$. All analyses were performed using STATISTICA 10.0 (Statsoft, Tulsa, OK, USA) software. Statistical procedures followed those described by Zar (1984).

\section{Results}

Jumping distance was influenced by background color only if the spiders approached leafhoppers $\left(t_{45}=6.79, p<0.001\right)$

but not if they approached caterpillars $\left(t_{37}=1.11, p=0.27\right)$ (Fig. 2). Leafhoppers were approached and attacked from about twice 
shorter the distance on light background than on dark background, while caterpillars were approached and attacked from similar distances on both backgrounds (Fig. 2).

Background color did not influence preyspecific behavior. The effect was irrespective of prey type. In approach to leafhoppers on either background there were no differences in the frequency of stalk $\left(G_{1 ; 47}=1.11, p=0.27\right)$. The spiders did not approach leafhoppers frontally and they did not temporarily release them after fang-piercing, therefore frontal approach and jump away were not recorded in the experiments with leafhoppers. Caterpillars were similarly approached and captured on light background and on dark background. Stalk was rare and occurred in similar frequencies on both backgrounds $\left(G_{1 ; 39}=0.001, p=0.97\right)$. Similar frequencies of frontal approach $\left(G_{1 ; 39}=0.13, p=0.71\right)$ and jump away $\left(G_{1 ; 39}=0.68, \quad p=0.41\right) \quad$ were observed on both backgrounds.

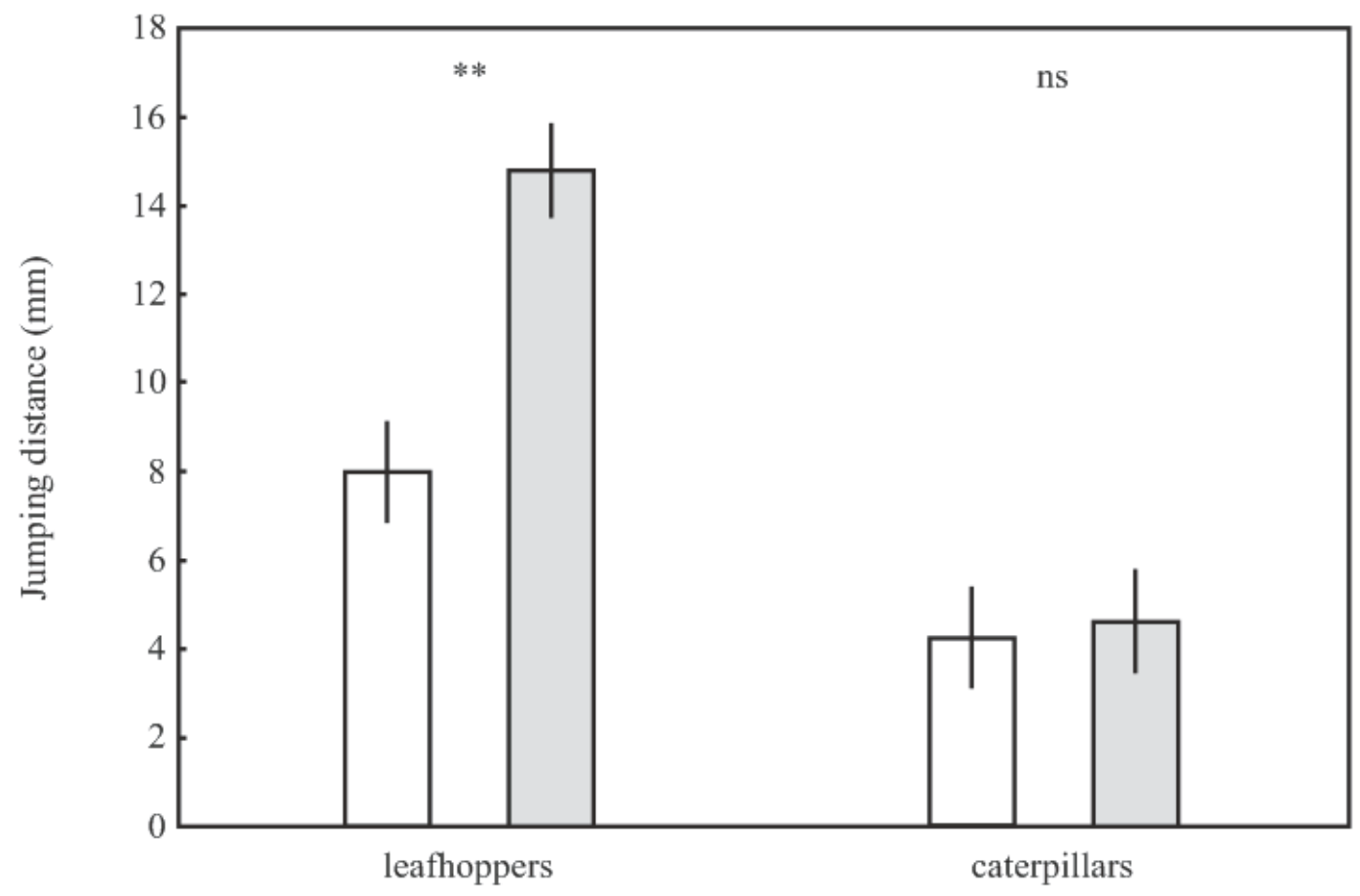

Prey type

Figure 2. Jumping distance of $Y$. arenarius on leafhoppers and caterpillars on light background (white bars) and dark background (grey bars). Bars are means; whiskers are $\pm 1.96 \mathrm{SE}$; double asterisk $(* *), p<0.001$; ns, lack of significant differences.

The predatory success of the spiders hunting leafhoppers on light background was significantly higher than on dark background $\left(G_{1 ; 47}=4.53, p=0.03\right)$. The spiders captured about $88 \%$ of leafhoppers on light background and about $61 \%$ of leafhoppers on dark background. All the prey that escaped did so

\section{Discussion}

The results of the tests provide evidence that $Y$. arenarius adapts its predatory behavior to prey type with respect to its own visibility to the prey. The change in the behavior after initial contact with the spider on the substrate, either during the initial strike or later, when the spider tried to subdue the prey. Predatory success of the spiders hunting caterpillars was $100 \%$ on both backgrounds, as the spiders always completed the strike and fang-pierced the caterpillars (Fig. 3).

occurred only with leafhoppers, the prey which can escape when it detects a predator, but the spiders did not change their behavior if approaching caterpillars, the prey that 
cannot efficiently escape. $Y$. arenarius and other jumping spiders were already known to use different prey-specific tactics against different prey. Alternative predatory tactics were commonly reported against the prey with high vs. low escape potential, such as flies, leafhoppers, grasshoppers possessing wings or jumping legs vs. insect larvae lacking such structures and the ability to escape efficiently (Edwards \& Jackson 1993; Bartos 2007). However, the situation when a jumping spider modifies its predatory behavior in response to an environmental factor only with some prey, but not the other prey, is rare and seems to be an example of an appreciable predatory complexity and behavioral plasticity rather unusual in invertebrates. It requires from the spider to visually detect the differences in background color, and to assess the potential of the observed prey to escape, possibly by identifying certain prey characteristics. Based on the information acquired, a certain preycapture technique is used.

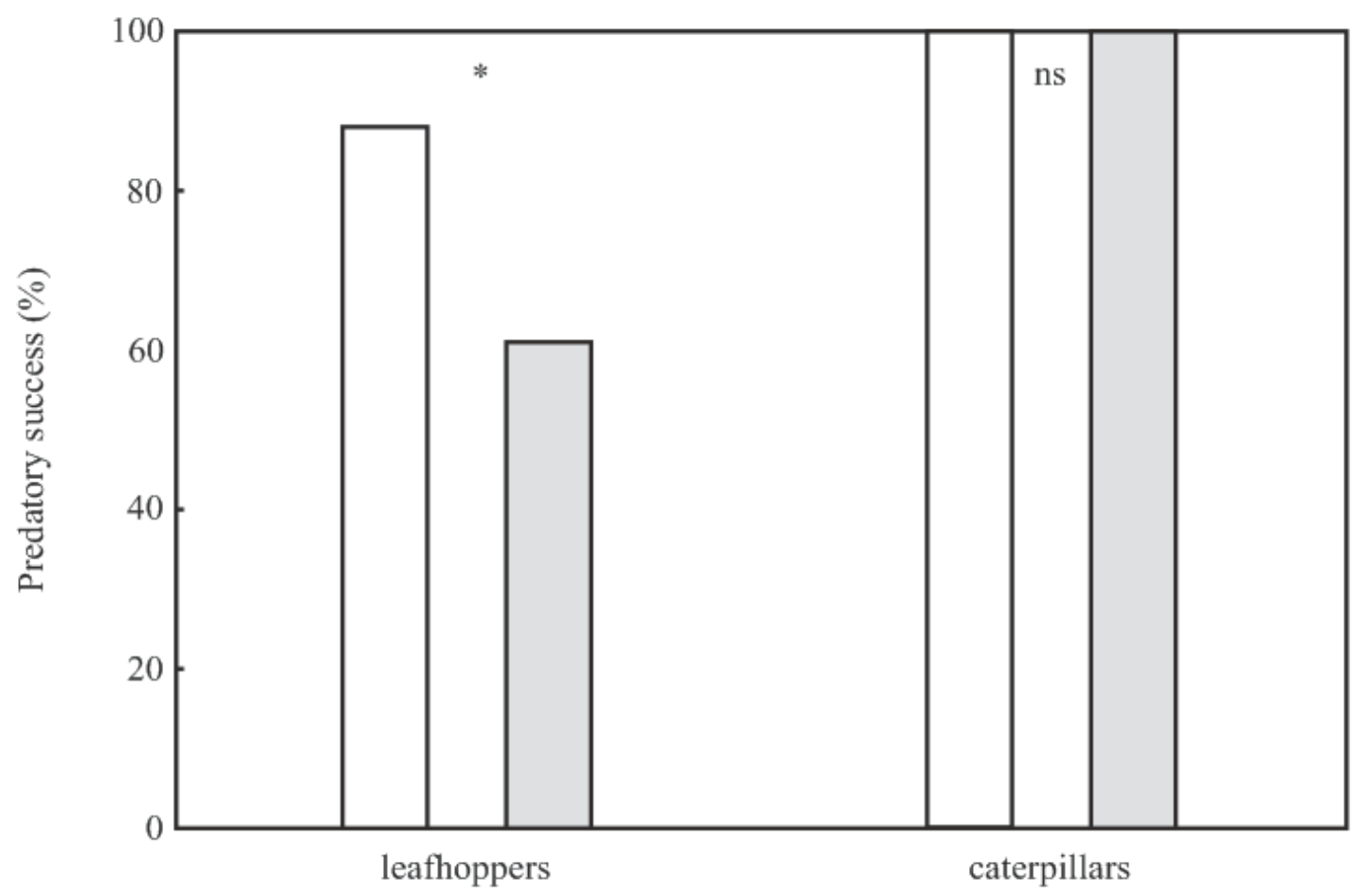

Prey type

Figure 3. Predatory success of juvenile $Y$. arenarius hunting leafhoppers and caterpillars on light background (white bars) and dark background (grey bars). Asterisk $(*), p<0.03$; ns, lack of significant differences.

This is one of a very few studies showing the influence of background color on stalking predator's decisions. Another study providing similar findings was on P. paykulli (Bear \& Hasson 1997). Prey items used in the tests with P. paykulli possessed similar escape abilities to those of the prey used with $Y$. arenarius. They were, however, from different insect orders. Some of the prey were anesthetized, and not live as in this study. Similar findings were also provided for several ambushing jumping spiders ( $\mathrm{Li}$ et al. 2003), which suggests that the jumping spiders' predatory flexibility involving the modification of basic predatory patterns in response to different visibility to their prey may not be a rare adaptation among jumping spiders.

The differences found between the tested groups draw our attention to the trade-offs between different types of risk the predator should take into account during prey capture (Bear \& Hasson 1997). Y. arenarius approaching leafhoppers on dark background increased jumping distance, which could reduce the risk of early detection. There was 
no case of prey escape before the strike, which suggests that the risk of early detection was very low, in fact at the same level as in the case of the spiders approaching on camouflaging background. The difference in predatory success between the two groups of spiders resulted probably from the risk of failure that appears in the late phase of predation, when the attack has already been launched. The risk is related to the lower precision of attack and lower ability to subdue the prey when the attack occurs from a longer distance. This is suggested by the fact that all the attacks occurred when the prey was still on the ground and before it started to escape. Even though early detection of the predator by the prey cannot be excluded it seems a rather unlikely explanation, as all the cases in which the prey was moving before the attack were excluded from the analysis.

Interestingly, even though all the tested prey-specific behaviors could theoretically influence the outcome of predatory encounter, the differences related to background color occurred only in the jumping distance, but not the other analyzed behaviors, such as stalk, frontal approach and jump away. Stalk, the behavior specific for the tactic used against the prey with high escape potential (Edwards \& Jackson 1993; Bartos 2007), seems to decrease the risk of early detection. A stalking spider moving slowly and using a characteristic choppy gait seems to reduce the risk of being noticed, at least on camouflaging background. On non-camouflaging background, however, a slowly moving spider has no concealment for a prolonged time, which should increase the risk of early detection or interference. In P. paykulli tested in similar conditions, stalk and other preyspecific behaviors were not analyzed, but the spider was reported to have approached faster to flies when non-camouflaged. This does not necessarily imply that the spiders stalk their prey less frequently when non-camouflaged, but may suggest some differences in predatory decisions between P. paykulli and $Y$. arenarius.

The other two analyzed behaviors, frontal approach and jump away, are specific for the tactic used against the prey with low escape potential. Frontal approach can generally increase the risk of early detection, but in the case of the prey that cannot escape it may have a negligible effect. In addition, frontal approach has never been observed to affect the caterpillar's velocity or the path of movement (Bartos unpubl. data). Therefore, it may not be perceived by caterpillars, even on dark background. This is especially likely for caterpillars in motion, when their own movement must notably impede the perception of the movement in their neighborhood.

The frequency of jump away analyzed in the study should, at least theoretically, affect the risk of interference by increasing the visibility of the spider and its prey. The spider hunting caterpillars usually leaves the wriggling caterpillar after initial venom injection and keeps at a distance until the venom paralyses the prey (Bartos 2007). If both the caterpillar and the spider are light in color, as in the experiment, the difference in their visibility on light vs. on dark background should result in different risks of both animals being seen on the backgrounds by a competitor or the spider's enemy. This could lead to any behaviors decreasing the risk of interference when non-camouflaged. The lack of differences between the camouflaging and the non-camouflaging background is therefore unexpected. There are no other studies to compare the results with. Bear and Hasson (1997) in their analyses had no data to discuss the risk of interference, but assumed that such a risk should occur. It seems intuitive that a predator trying to subdue a prey on a non-camouflaging background should suffer a higher risk of being noticed by its enemy than hunting on camouflaging background. The risk may, however, primarily depend on their major enemies, particularly their methods of searching the prey, sensory abilities to detect the prey and the intensity of their pressure. The majority of bare areas of sandy habitats $Y$. arearius dwells in are lacking day-active vertebrate predators or other predators with good eyesight. Major predators for the spiders 
are their conspecifics, ant-lions and several ant species, other enemies, such as tiger beetles and robber flies are rather infrequent. Long-term field observations carried out for over a decade (Bartos unpubl. data) suggest that predatory pressure is generally low in the case of $Y$. arenarius, which may, at least partially, explain the lack of differences between the frequencies of jump away on the tested backgrounds.

\section{Acknowledgments}

This research was supported by the Polish Ministry of Scientific Research and Information Technology (grant number SCSR 3P04F05822) and the University of Lodz.

\section{References}

Bartos, M. 2004. The prey of Yllenus arenarius (Araneae, Salticidae). Bulletin of the British Arachnological Society, 13: 83-85.

Bartos, M. 2005. The life history of Yllenus arenarius (Araneae, Salticidae) - evidence for sympatric populations isolated by the year of maturation. Journal of Arachnology, 33: 222-229.

Bartos, M. 2007. Hunting prey with different escape potentials - alternative predatory tactics in a dunedwelling salticid. Journal of Arachnology, 35: 499509.

Bartos, M. 2008. Alternative predatory tactics in a juvenile jumping spider. Journal of Arachnology, 36: 300-305.

Bartos, M. 2011. Partial dietary separation between coexisting cohorts of Yllenus arenarius (Araneae: Salticidae). Journal of Arachnology, 39: 230-235.

Bear, A. \& Hasson, O. 1997. The predatory response of

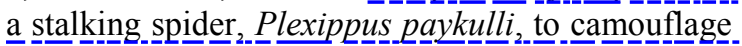
and prey type. Animal Behaviour, 54: 993-998.

Blest, A.D., Hardie, R.C., McIntyre, P. \& Williams, D.S. 1981. The spectral sensitivities of identified receptors and the function of retinal tiering in the principal eyes of a jumping spider. Journal of Comparative Physiology A, 145: 227-239.

Blest, A.D., O'Carrol, D.C. \& Carter, M. 1990. Comparative ultrastructure of layer I receptor mosaics in principal eyes of jumping spiders: the evolution of regular arrays of light guides. Cell and Tissue Research, 262: 445-460.

Briscoe, A.D. \& Chittka, L. 2001. The evolution of color vision in insects. Annual Revue of Entomology, 46: 471-510.

Edwards, G.B. \& Jackson, R.R. 1993. Use of preyspecific predatory behaviour by North American jumping spiders (Araneae, Salticidae) of the genus Phidippus. Journal of Zoology, 229: 709-716.

Forster, L.M. 1977. A qualitative analysis of hunting. behaviour in jumping spiders (Araneae: Salticidae). New Zealand Journal of Zoology, 4: 51-62.

Forster, L.M. 1982. Vision and prey-catching strategies in jumping spiders. American Scientist, 70: 165175 .

Harland, D.P. \& Jackson, R.R. 2004. Portia Perceptions: the Umwelt of an araneophagic jumping spider. In: Prete F.R. (ed.), Complex worlds from simpler nervous systems. MIT Press, Cambridge, pp. 5-40.

Jackson, R.R \& Carter, C.M. 2001. Geographic variation in reliance on trial-and-error signal derivation by Portia labiata, an araneophagic jumping spider from the Philippines. Journal of Insect Behavior, 14: 799-827.

Jakob, E., Skow, C. \& Long, S. 2011. Plasticity, learning and cognition. In: Herberstein M.E. (ed.), Spider behaviour: flexibility and versatility. Cambridge University Press, New York pp. 307347.

Jackson, R.R. \& Nelson, X. 2012. Attending to detail by communal spider-eating spiders. Animal Cognition, 15: 461-471.

Land, M.F. 1969a. Structure of the retinae of the principal eyes of jumping spiders (Salticidae: Dendryphantinae) in relation to visual optics. Journal of Experimental Biology, 51: 443-470.

Land, M.F. 1969b. Movements of the retinae of jumping spiders (Salticidae: Dendryphantinae) in response to visual stimuli. Journal of Experimental Biology, 51: 471-493.

Land, M.F. 1972. Mechanisms of orientation and pattern recognition by jumping spiders (Salticidae). In: Wehner R. (ed.), Information processing in the visual systems of arthropods. Springer, Berlin, pp. 231-247.

Land, M.F. 1985. Fields of view of the eyes of primitive jumping spiders. Journal of Experimental Biology, 119: 381-384.

Li, D., Jackson, R.R., \& Barrion, A. 1999. Parental and predatory behaviour of Scytodes sp., an araneophagic spitting spider (Araneae: Scytodidae) from the Philippines. Journal of Zoology, London, 247: 293-310.

Li, D. \& Jackson, R.R. 2003. A predator's preference for egg-carrying prey: a novel cost of parental care. Behavioral Ecology and Sociobiology, 55: 129136.

Li, D., Jackson, R.R. \& Lim, M.L.M. 2003. Influence of background and prey orientation on an ambushing predator's decisions. Behaviour, 140: 739-764.

Li, J., Lim, M.L.M., Zhang, Z., Liu, Q., Liu, F., Chen, J. \& Li, D. 2008. Sexual dichromatism and male 
colour morph in ultraviolet-B reflectance in two populations of the jumping spider Phintella vittata (Araneae: Salticidae) from tropical China. Biological Journal of the Linnean Society, 94: 720.

Nakamura, T. \& Yamashita, S. 2000. Learning and discrimination of colored papers in jumping spiders (Araneae, Salticidae). Journal of Comparative Physiology A, 186: 897-901.

Nelson, X.J. \& Jackson, R.R. 2011. Flexibility in the foraging strategies of spiders. In: Herberstein M.E. (ed.), Spider behaviour: flexibility and versatility, Cambridge University Press, New York, pp. 31-56.

Nelson, X.J. \& Jackson, R.R. 2012a. Fine-tuning of vision-based prey-choice decisions by a predator that targets malaria vectors. Journal of Arachnology, 40: 23-33.

Nelson, X.J. \& Jackson, R.R. 2012b. The discerning predator: decision rules underlying prey classification by a mosquito-eating jumping spider. Journal of Experimental Biology, 215: 2255-2261.

Peaslee, A.G. \& Wilson, G. 1989. Spectral sensitivity in jumping spiders (Araneae, Salticidae). Journal of Comparative Physiology A, 164: 359-63.

Williams, D. \& McIntyre, P. 1980. The principal eyes of a jumping spider have a telephoto component. Nature, 288: 578-580.

Yamashita, S. \& Tateda, H. 1976. Spectral Sensitivities of Jumping Spider Eyes. Journal of Comparative Physiology A, 105: 29-41.

Zar, J.H. 1984. Biostatistical Analysis. 2nd edition. Prentice-Hall, New Jersey.

Zurek, D. \& Nelson, X.J. 2012. Saccadic tracking of targets mediated by the anterior-lateral eyes of jumping spiders. Journal of Comparative Physiology A: Neuroethology, Sensory, Neural, and Behavioral Physiology, 198: 411-417. 